\title{
A holographic bound for D3-brane
}

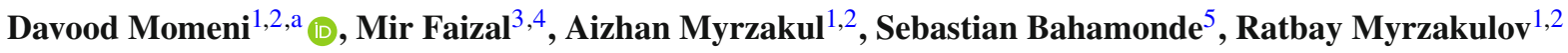 \\ ${ }^{1}$ Eurasian International Center for Theoretical Physics, Eurasian National University, Astana 010008, Kazakhstan \\ 2 Department of General Theoretical Physics, Eurasian National University, Astana 010008, Kazakhstan \\ ${ }^{3}$ Irving K. Barber School of Arts and Sciences, University of British Columbia-Okanagan, 3333 University Way, Kelowna, BC V1V 1V7, Canada \\ ${ }^{4}$ Department of Physics and Astronomy, University of Lethbridge, Lethbridge, AB T1K 3M4, Canada \\ ${ }^{5}$ Department of Mathematics, University College London, Gower Street, London WC1E 6BT, UK
}

Received: 6 April 2017 / Accepted: 29 May 2017 / Published online: 13 June 2017

(C) The Author(s) 2017. This article is an open access publication

\begin{abstract}
In this paper, we will regularize the holographic entanglement entropy, holographic complexity and fidelity susceptibility for a configuration of D3-branes. We will also study the regularization of the holographic complexity from the action for a configuration of D3-branes. It will be demonstrated that for a spherical shell of D3-branes the regularized holographic complexity is always greater than or equal to the regularized fidelity susceptibility. Furthermore, we will also demonstrate that the regularized holographic complexity is related to the regularized holographic entanglement entropy for this system. Thus, we will obtain a holographic bound involving regularized holographic complexity, regularized holographic entanglement entropy and regularized fidelity susceptibility of a configuration of D3-brane. We will also discuss a bound for regularized holographic complexity from action, for a D3-brane configuration.
\end{abstract}

In this paper, we will analyze the relation between the holographic complexity, holographic entanglement entropy and fidelity susceptibility for a spherical shell of D3-branes. We shall also analyze the holographic complexity from the action for a configuration of D3-branes. These quantities will be geometrically calculated using the bulk geometry, and the results thus obtained will be used to demonstrate the existence of a holographic bound for configurations of D3-branes. It may be noted that there is a close relation between the geometric configuration involving D3-branes and quantum informational systems [1]. It is well known that D3-branes can be analyzed as a real three-qubit state [2]. This is done using the configurations of intersecting D3branes, wrapping around the six compact dimensions. The $T^{6}$ provides the microscopic string-theoretic interpretation of the charges. The most general real three-qubit state can be parameterized by four real numbers and an angle, and the most general STU black hole can be described by four

a e-mail: d.momeni@yahoo.com
D3-branes intersecting at an angle. Thus, it is possible to represent a three-qubit state by D3-branes. A system of D3branes has been used to holographically analyze the quantum Hall effect, as a system of D3-D7-branes has been used to obtain the Hall conductivity and the topological entanglement entropy for the quantum Hall effect [3]. The mutual information between two spherical regions in $\mathcal{N}=4$ superYang-Mills theory dual to type IIB string theory on $\mathrm{AdS}_{5} \times S^{5}$ space has been analyzed using correlators of surface operators [4]. Such a surface operator corresponds to having a D3-brane in $\mathrm{AdS}_{5} \times S^{5}$ space ending on the boundary along the prescribed surface. This construction relies on the strong analogies between the twist field operators used for the computation of the entanglement entropy, and the disorder-like surface operators in gauge theories. A configuration of D3branes and D7-branes with a non-trivial worldvolume gauge field on the D7-branes has also been used to holographically analyze a new form of quantum liquid, with certain properties resembling a Fermi liquid [5] The holographic entanglement entropy of an infinite strip subsystem on the asymptotic AdS boundary has been used as a probe to study the thermodynamic instabilities of planar R-charged black holes and their dual field theories [6]. This was done using spinning D3-branes with one non-vanishing angular momentum. It was demonstrated that the holographic entanglement entropy exhibits the thermodynamic instability associated with the divergence of the specific heat. When the width of the strip was large enough, the finite part of the holographic entanglement entropy as a function of the temperature resembles the thermal entropy. However, as the width become smaller, the two entropies behave differently. It was also observed that below a critical value for the width of the strip, the finite part of the holographic entanglement entropy as a function of the temperature develops a self-intersection.

Thus, there is a well established relation between different D3-brane configurations and information theoretical 
processes. Thus, it would be interesting to analyze different information theoretical quantities for a configuration of D3branes. It may be noted that entropy is one of the most important quantities in information theoretical processes. This is because entropy measures the loss of information during a process. It may be noted that the maximum entropy of a region of space scales with its area, and this observation has been motivated from the physics of black holes. This observation has led to the development of the holographic principle $[7,8]$. The holographic principle equates the degrees of freedom in a region of space to the degrees of freedom on the boundary surrounding that region of space. The AdS/CFT correspondence is a concrete realizations of the holographic principle [9-11], and it relates the string theory in AdS to a superconformal field theory on the boundary of that AdS. The AdS/CFT correspondence in turn can be used to holographically obtain the entanglement entropy of a boundary field theory. The holographic entanglement entropy of a conformal field theory on the boundary of an AdS solution is dual to the area of a minimal surface defined in the bulk. Thus, for a subsystem as $A$, we can define $\gamma_{A}$ as the $(d-1)$-minimal surface extended into the AdS bulk, with the boundary $\partial A$. Now using this subsystem, the holographic entanglement entropy can be expressed as $[12,13]$

$S_{A}=\frac{\mathcal{A}\left(\gamma_{A}\right)}{4 G_{d+1}}$,

where $G$ is the gravitational constant for the bulk AdS and $\mathcal{A}\left(\gamma_{A}\right)$ is the area of the minimal surface. Even though this quantity is divergent, it can be regularized $[14,15]$. The holographic entanglement entropy can be regularized by subtracting the contribution of the background AdS spacetime from the deformation of the AdS spacetime. Thus, for the system studied in this paper, let $\mathcal{A}\left[\mathrm{D} 3\left(\gamma_{A}\right)\right]$ be the contribution of a D3-brane shell and $\mathcal{A}\left[\operatorname{AdS}\left(\gamma_{A}\right)\right]$ be contribution of the background AdS spacetime, then the regularized holographic entanglement entropy will be given by

$\Delta S_{A}=\frac{\mathcal{A}\left[\mathrm{D} 3\left(\gamma_{A}\right)\right]-\mathcal{A}\left[\operatorname{AdS}\left(\gamma_{A}\right)\right]}{4 G_{d+1}}$.

In this paper, we will use this regularized holographic entanglement entropy.

The entropy measures the loss of information during a process. However, it is also important to know how easy it is for an observer to extract this information. The complexity quantified this idea relating it to the difficulty to extract information. It is expected that complexity is another fundamental physical quantify, as it is an important quantity in information theory, and the laws of physics can be represented in terms of informational theoretical processes. In fact, complexity has been used in condensed matter systems $[16,17]$ and molecular physics $[18,19]$. Complexity is also important in black hole physics, as it has been proposed that even though the information may not be ideally lost during the evaporation of a black hole, it would be effectively lost during the evaporation of a black hole. This is because it would become impossible to reconstruct it from the Hawking radiation [20]. It has been proposed that the complexity can be obtained holographically as a quantity dual to the volume of a codimension one time slice in the anti-de Sitter (AdS) [21-23] case,

Complexity $=\frac{V}{8 \pi R G_{d+1}}$,

where $R$ and $V$ are the radius of the curvature and the volume in the AdS bulk.

As it is possible to define the volume in different ways in the AdS, different proposals for the complexity have been made. If this volume is defined to be the maximum volume in AdS which ends on the time slice at the AdS boundary, $V=V\left(\Sigma_{\max }\right)$, then the complexity corresponds to the fidelity susceptibility $\chi_{F}$ of the boundary conformal field theory [24]. This quantity diverges [25]. However, we will regularize it by subtracting the contribution of the background AdS spacetime from the contribution of the deformation of AdS spacetime. So, let $V\left[\mathrm{D} 3\left(\Sigma_{\max }\right)\right]$ be the contribution of a D3-brane shell and $V\left[\operatorname{AdS}\left(\Sigma_{\max }\right)\right]$ be the contribution of the background AdS spacetime, then we can write the regularized fidelity susceptibility as

$\Delta \chi_{F}=\frac{V\left[\mathrm{D} 3\left(\Sigma_{\max }\right)\right]-V\left[\operatorname{AdS}\left(\Sigma_{\max }\right)\right]}{4 G_{d+1}}$.

It is also possible to use a subsystem $A$ (with its complement), to define a volume in the AdS case as $V=V\left(\gamma_{A}\right)$. This is the volume which is enclosed by the minimal surface used to calculate the holographic entanglement entropy [26]. Thus, using $V=V\left(\gamma_{A}\right)$, we obtain the holographic complexity as $\mathcal{C}_{A}$. As we want to differentiate between these two cases, we shall call this quantity, defined by $V=V\left(\Sigma_{\max }\right)$, the fidelity susceptibility, and the quantity defined by $V=V\left(\gamma_{A}\right)$ the holographic complexity. The holographic complexity diverges [25]. We will regularize it by subtracting the contributions of the background AdS from the deformation of the AdS spacetime. Now if $V\left[\mathrm{D} 3\left(\gamma_{A}\right)\right]$ is the contribution of a D3-brane shell and $V\left[\operatorname{AdS}\left(\gamma_{A}\right)\right]$ is the contribution of the background AdS spacetime, then we can write the regularized holographic complexity as

$\Delta C_{A}=\frac{V\left[\mathrm{D} 3\left(\gamma_{A}\right)\right]-V\left[\operatorname{AdS}\left(\gamma_{A}\right)\right]}{4 G_{d+1}}$.

It may be noted that there is a different proposal for calculating the holographic complexity of a system using the 
action $[27,28]$. According to this proposal, the holographic complexity of a system can be related to the bulk action evaluated on the Wheeler-DeWitt patch,

$\mathcal{C}_{W}=\frac{A(W)}{\pi \hbar}$,

where $A(W)$ is the action evaluated on the Wheeler-DeWitt patch $W$, with a suitable boundary time. To differentiate it from the holographic complexity calculated from the volume $\mathcal{C}$, we shall call this quantity the "holographic complexity from action", and denote it by $\mathcal{C}_{W}$ (as it has been calculated on a Wheeler-DeWitt patch). This quantity also diverges [29]. We shall regularize it by subtracting the contributions of the AdS spacetime from the contributions of the deformation of the AdS spacetime. So, if $A[\mathrm{D} 3(W)]$ is the contribution of a D3-brane shell and $A[\operatorname{AdS}(W)]$ is the contribution of the background AdS spacetime, then we can write the regularized holographic complexity from the action

$\Delta \mathcal{C}_{W}=\frac{A[\mathrm{D} 3(W)]-A[\operatorname{AdS}(W)]}{\pi \hbar}$.

It may be noted that this proposal is very different from the other proposals to calculate the complexity of a boundary theory. This difference occurs as there are differences in the definition of complexity for a boundary field theory. So, this proposal cannot be directly related to the proposals where the complexity can be calculated from the volume of a geometry. In fact, it is possible to have the same volume for two theories with different field content. In this paper, we will first use calculate a bound for the D3-brane geometries using the volume of a shell of D3-branes. Then we shall calculate a different holographic bound for a configuration of D3-branes using the action of this system.

In this paper, we will analyze a specific configuration of D3-branes and discuss the behavior of these regularized information theoretical quantities for it. It is possible to use a static gauge and write the bosonic part of the action for such a system in $\mathrm{AdS}_{5} \times S^{5}$ background as [35]

$$
\begin{aligned}
A= & \frac{1}{2 \pi g_{s} k^{2}} \int\left(\sqrt{-h}-\sqrt{-\operatorname{det}\left(G_{\mu \nu}+k F_{\mu \nu}\right)}\right) d^{4} x \\
& +\frac{\chi}{8 \pi} \int F \wedge F,
\end{aligned}
$$

where $k=\sqrt{g_{s} N / \pi}$ and

$G_{\mu \nu}=h_{\mu \nu}+k^{2} \frac{\partial_{\mu} \phi^{I} \partial_{\nu} \phi^{I}}{\phi^{2}}$.

Here $h_{\mu \nu}=\phi^{2} \eta_{\mu \nu}, h=\operatorname{det} h_{\mu \nu}$, with $\eta_{\mu \nu}$ being the four dimensional Minkowski metric. Thus, we can write $\sqrt{-h}=\phi^{4}$, where $\phi^{2}=\sum\left(\phi^{I}\right)^{2}$, and $\phi^{I}$ are six scalar fields corresponding to the six dimensions transverse to the D3-brane geometry. It may be noted that the $\int F \wedge F$ term only contributes to the magnetically charged configurations. The D3-brane can be placed at a fixed position on $S^{5}$, such that the five scalar fields corresponding to the $S^{5}$ geometry will not have any contribution. We shall consider the spherically symmetrical static solutions, centered at $r=0$, for this geometry. So, the electric field $\mathbf{E}$ and the magnetic fields $\mathbf{B}$ will only have radial components, which we shall denote by $E$ and $B$. So, all fields of this system are only functions of the radial coordinate $r, E(r), B(r), \phi(r)$. Thus, we can write $\operatorname{det}\left(-G_{\mu \nu}\right)=\phi^{6} G_{r r}=\phi^{6}\left[\phi^{2}+\gamma^{2}\left(\phi^{\prime} / \phi\right)^{2}\right]$, and

$-\operatorname{det}\left(G_{\mu \nu}+\gamma F_{\mu \nu}\right)=\phi^{6}\left(G_{r r}-\frac{\gamma^{2} E^{2}}{\phi^{2}}\right)\left(1+\frac{\gamma^{2} B^{2}}{\phi^{4}}\right)$.

So, the Lagrangian density for this system can be written as

$$
\begin{aligned}
\mathcal{L}= & \frac{1}{\gamma^{2}} \phi^{4}\left(1-\sqrt{\left(1+\frac{\gamma^{2}\left[\left(\phi^{\prime}\right)^{2}-E^{2}\right]}{\phi^{4}}\right)\left(1+\frac{\gamma^{2} B^{2}}{\phi^{4}}\right)}\right) \\
& +g_{s} \chi B E,
\end{aligned}
$$

where $\gamma=\sqrt{\frac{N}{2 \pi^{2}}}=R^{2} \sqrt{T_{\mathrm{D} 3}}, T_{\mathrm{D} 3}$ is D3-brane tension. There are two BPS solutions for this geometry, $\phi_{ \pm}=\mu \pm$ $Q / r$. The probe $\mathrm{D} 3$-brane solution discussed here describes a BIon like spike (either up to the $\mathrm{AdS}_{5}$ boundary or down to the Poincaré horizon, depending on the sign in $\phi_{ \pm}$). This solution also breaks the translational symmetry in the field theory, and it preserves the rotational invariance.

It is also possible to analyze a probe D3-brane with $Q=$ $0, E=0$, and $B=0$. Now we will analyze such a specific solution representing a D3-brane configuration, and analyze these quantities for that specific geometric configuration. It is possible to study such a D3-brane shell. The metric for the near horizon geometry of D3-brane shell is given by [30]

$$
\mathrm{d} s^{2}=\frac{R^{2}}{z^{2} h(z)}\left(\sum_{\mu=0}^{3} \mathrm{~d} x_{\mu} \mathrm{d} x^{\mu}\right)+R^{2} h(z)\left(\frac{\mathrm{d} z^{2}}{z^{2}}+d \Omega_{5}\right),
$$

where the function $h(z)$ is defined as

$h(z)=\left\{\begin{array}{ll}1, & z \leq z_{0} \\ \left(\frac{z_{0}}{z}\right)^{2}, & z \geq z_{0}\end{array}\right.$.

For this geometry, the entangled region is a strip with width $\ell$ in the D3-brane shell defined by the embedding $A=\{x=$ $x(z), t=0\}$. The area functional can be expressed as

$$
\mathcal{A}\left(\gamma_{A}\right)=2 \pi^{3} R^{8} L^{2} \int_{0}^{z_{*}} \frac{h(z) \sqrt{x^{\prime}(z)^{2}+h(z)^{2}}}{z^{3}} \mathrm{~d} z,
$$


where $x^{\prime}\left(z_{*}\right)=\infty$. The Euler-Lagrange equation for $x(z)$ has the following form:

$$
\frac{x^{\prime}(z)}{\sqrt{x^{\prime}(z)^{2}+h(z)^{2}}}=\frac{h\left(z_{*}\right)}{h(z)}\left(\frac{z}{z_{*}}\right)^{3} .
$$

The total length can be obtained by

$\ell=2 \int_{0}^{z_{*}} \mathrm{~d} z h(z)\left[\frac{\frac{h\left(z_{*}\right)}{h(z)}\left(\frac{z}{z_{*}}\right)^{3}}{\sqrt{1-\left(\frac{h\left(z_{*}\right)}{h(z)}\left(\frac{z}{z_{*}}\right)^{3}\right)^{2}}}\right]^{1 / 2}$.

We can also write the volume $V\left(\gamma_{A}\right)$ as

$V\left(\gamma_{A}\right)=2 \pi^{3} R^{9} L^{2} \int_{0}^{z_{*}} \frac{h(z)^{3 / 2}}{z^{4}} x(z) \mathrm{d} z$.

We can solve Eq. (15) exactly and obtain

$x(z)=\left\{\begin{array}{l}C_{1}+\int \frac{h\left(z_{*}\right) z^{3}}{\sqrt{-h\left(z_{*}\right)^{2} z^{6}+z_{0}}} \mathrm{~d} z, \quad z \leq z_{0}, \\ C_{2}+\int \frac{h\left(z_{*}\right) z^{3} z 0^{2}}{\sqrt{-z^{10} h\left(z_{*}\right)^{2}+z_{0} 10}} \mathrm{~d} z, \quad z \geq z_{0} .\end{array}\right.$,

where $C_{1}$ and $C_{2}$ are integration constants. The maximal volume, which is related to the fidelity susceptibility, is given by

$$
V\left(\Sigma_{\max }\right)=2 \pi^{3} R^{9} L^{3} \int_{0}^{z_{\infty}} \frac{h(z)^{3 / 2}}{z^{4}} \mathrm{~d} z .
$$

Now we will use $h(z)$, and split the integral into two parts, $\int_{0}^{z_{\infty}}=\int_{0}^{z_{0}}+\int_{z_{0}}^{z_{\infty}}$, to obtain

$V\left(\Sigma_{\max }\right)=\frac{-\pi^{3} R^{9} L^{3}}{3} \frac{z_{0}{ }^{6}+z_{\infty}{ }^{6}}{z_{\infty} z_{0}{ }^{3}}$.

It may be noted that by setting $C_{1}=C_{2}=L$, the difference of the volumes (17) and (20) is given by

$V\left(\gamma_{A}\right)-V\left(\Sigma_{\max }\right)= \begin{cases}\int_{0}^{z_{0}} \frac{h\left(z_{*}\right) z^{3}}{\sqrt{-h\left(z_{*}\right)^{2} z^{6}+z_{0}}} \mathrm{~d} z, & z \leq z_{0}, \\ \int_{z_{0}}^{z_{\infty}} \frac{h\left(z_{*}\right) z^{3} z_{0}}{\sqrt{-z^{10} h\left(z_{*}\right)^{2}+z_{0}{ }^{10}}} \mathrm{~d} z, \quad z \geq z_{0} .\end{cases}$

Since $h\left(z_{*}\right)>0$, we can express this as

$V\left(\gamma_{A}\right)-V\left(\Sigma_{\max }\right) \geq \begin{cases}\frac{z_{0} h\left(z_{*}\right)}{4}, & z \leq z_{0}, \\ \frac{h\left(z_{*}\right)}{4 z_{0}{ }^{3}}\left(z_{\infty}^{4}-z_{0}^{4}\right), & z \geq z_{0} .\end{cases}$

So, for a D3-brane, we obtain a relation between $V\left(\gamma_{A}\right)$ and $V\left(\Sigma_{\max }\right), V\left(\gamma_{A}\right)-V\left(\Sigma_{\max }\right) \geq 0$. However, as the holographic complexity and fidelity susceptibility for a system are obtained using $V\left(\gamma_{A}\right)$ and $V\left(\Sigma_{\max }\right)$, we obtain the following bound for a D3-brane:

$\Delta \mathcal{C}_{A} \geq \Delta \chi_{F}$

So, we have demonstrated that for a D3-brane the holographic complexity is always greater than or equal to the fidelity susceptibility. This was expected, as the fidelity susceptibility is calculated using the maximum volume in the bulk, and the holographic complexity is only calculated for a subsystem.

It is also possible to demonstrate that a relation exists between the holographic complexity and the entanglement entropy of D3-brane. To obtain this relation between the holographic complexity and entanglement entropy of a D3-brane, we note that $\Delta S_{A}$ is given by

$\Delta S_{A}=\frac{\pi^{3} R^{8} L^{2}}{2 G} \int_{0}^{z_{*}}\left(\frac{h(z) \sqrt{x^{\prime}(z)^{2}+h(z)^{2}}-\sqrt{x_{\mathrm{AdS}}^{\prime}(z)^{2}+1}}{z^{3}}\right) \mathrm{d} z$,

and $\Delta \mathcal{C}_{A}$ is given by

$\Delta \mathcal{C}_{A}=\frac{\pi^{2} R^{8} L^{2}}{4 G} \int_{0}^{z_{*}}\left(\frac{h(z)^{3 / 2} x(z)-x_{\mathrm{AdS}}(z)}{z^{4}}\right) \mathrm{d} z$,

where

$x_{\text {AdS }}(z)=\left\{\begin{array}{l}C_{1}^{\prime}+\int \frac{z^{3}}{\sqrt{-z^{6}+z_{0}^{6}}} \mathrm{~d} z, \quad z \leq z_{0}, \\ C_{2}^{\prime}+\int \frac{z^{3} z_{0}^{2}}{\sqrt{-z^{10}+z_{0}{ }^{10}}} \mathrm{~d} z, \quad z \geq z_{0},\end{array}\right.$

because $z \sim 0$ is near the AdS boundary limit. So, now as $z_{*}<z_{0}$, we obtain

$\Delta S_{A} \approx \frac{\pi^{3} R^{8} L^{2}}{z_{*}^{2} G}$,

$\Delta \mathcal{C}_{A} \approx-\frac{3}{4} \frac{\pi^{2} R^{8} L^{2}}{z_{*}^{3} G}\left(C_{1}-C_{1}^{\prime}\right)$.

The total length of this system can be written as

$$
l \approx \frac{4 \sqrt{h\left(z_{*}\right)} z_{*}}{5} .
$$

By defining the effective holographic temperature $T_{\text {ent }} \sim$ $l^{-1}$, we obtain the relation between the holographic complexity and the holographic entanglement entropy,

$$
\Delta \mathcal{C}_{A}=\frac{c \Delta S_{A}}{T_{\mathrm{ent}} R}
$$

where $c$ is given by

$$
c=\frac{3}{5} \frac{C_{1}^{\prime}-C_{1}}{\pi} \frac{R}{c_{T}} \text {. }
$$

Here $c_{T}$ is the proportionality coefficient in the definition of the $T_{\text {ent }}$ [31,32], and $C_{1}^{\prime}, C_{1}$ are integration constants. As the only dependence of $c$ on the geometry is from the AdS radius $R$, the value of the coefficient $c$ does not depend on the specific deformation of the AdS geometry, and so it cannot depend on the specific configuration of the D3-branes. It may be noted that this bound can also be used to understand the meaning of the holographic complexity for a boundary theory, as all the other quantities are defined for boundary theory, and thus this relation can be used to understand the behavior of the holographic complexity for the boundary theory. 
Thus, we have obtained a relation between the holographic complexity and holographic entanglement entropy for a D3brane. However, as the holographic complexity is also related to the fidelity susceptibility, we obtain the following holographic bound for a D3-brane:

$\frac{c \Delta S_{A}}{T_{\mathrm{ent}} R}=\Delta \mathcal{C}_{A} \geq \Delta \chi_{F}$.

It may be noted that a bound on the holographic entanglement entropy for a fixed effective holographic temperature can be translated into a bound on the holographic complexity, and this in turn can be related to a bound on the fidelity susceptibility. So, we have obtained a relation between the holographic complexity, holographic entanglement entropy and fidelity susceptibility for a D3-brane. The holographic entanglement entropy is directly proportional to the holographic complexity, when the effective holographic temperature is fixed. Furthermore, the holographic complexity is always greater than or equal to the fidelity susceptibility, so the fidelity susceptibility can also be related to the holographic entanglement entropy.

As it has recently been proposed that the holographic entanglement entropy can be calculated from the action evaluated at a Wheeler-DeWitt patch [27,28], we shall now calculate the holographic complexity from the action for this D3-brane configuration. It may be noted that it is expected that the holographic complexity from the action will satisfy the bound [33]. This bound has been tested for different AdS black hole geometries [27, 28,34], and we will test it for a D3brane configuration. Now the holographic complexity from the action for this D3-brane configuration can be obtained by evaluating the bulk action on the Wheeler-DeWitt patch. The full type IIB action cannot be used for such a calculation as no action is known for the self-dual five form, which exists in the full theory. So, we will evaluate the probe D3-brane action on the Wheeler-DeWitt patch, and not use the full type IIB action. In fact, this solution will depend on $Q$, which exists in the probe solution, and not the domain wall solution. So, this only represents the probe D3-brane action on the WheelerDeWitt patch. Now we will calculate the contributions of the probe to the complexity from the action. As this quantity is divergent, we will also subtract the background AdS contribution from this quantity. Thus, the regularized holographic complexity from the action, for this D3-brane contribution, can be written as

$$
\begin{aligned}
& \Delta \mathcal{C}_{W}=\frac{R^{10} V_{3} \Omega_{5}}{\pi \hbar}\left(\frac{1}{256} \frac{\gamma^{6} Q^{8}\left(z_{0}{ }^{16}-\epsilon^{16}\right)}{\mu^{12} r_{0}{ }^{16}}-\frac{1}{17} \frac{\gamma^{6} Q^{9} z_{0}{ }^{17}}{\mu^{13} r_{0}{ }^{17}}\right. \\
& +\frac{17}{36} \frac{\gamma^{6} Q^{10} z_{0}{ }^{18}}{\mu^{14} r_{0}{ }^{18}}-\frac{1}{4} \frac{Q^{2} z_{0}^{2}\left(z_{\infty}^{2}-z_{0}^{2}\right)}{r_{0}{ }^{4}}
\end{aligned}
$$

$$
\begin{aligned}
& -\frac{1}{6}\left(-\frac{35}{2} \frac{Q^{6} \gamma^{2}}{\mu^{4} r_{0}{ }^{4}}+\mu^{4} r_{0}^{4}\left(\frac{35}{2} \frac{Q^{6} \gamma^{2}}{\mu^{8} r_{0}{ }^{8}}-\frac{1}{8} \frac{\gamma^{4} Q^{4}}{\mu^{8} r_{0}{ }^{8}}\right)\right) \\
& \left.\times z_{0}^{2}\left(z_{\infty}^{6}-z_{0}^{6}\right) r_{0}{ }^{-4} \gamma^{-2}\right),
\end{aligned}
$$

where $\epsilon$ is an IR cutoff and $z_{\infty}$ is the replacement for a UV cutoff. It may be noted that unlike the holographic complexity or fidelity susceptibility, this holographic complexity from the action does not only depend on the geometry, but on details of the field content of the theory. Thus, it cannot be related to the holographic complexity, or fidelity susceptibility, or even the holographic entanglement entropy in a direct way. This is because these quantities are purely geometric quantities. The main reason for this difference is that unlike the entropy, there is an ambiguity in the definition of the complexity, and thus many alternative proposals have been made to define the complexity of a boundary theory. Thus, we cannot relate the holographic complexity from the action to those other purely geometric quantities. However, we can calculate a different kind of bound for this holographic complexity from the action. Thus, using the Poincaré coordinate $z$, such that $z \equiv \frac{r_{0}}{r}, r_{0}=\frac{Q}{v}$, we obtain

$$
\begin{aligned}
& \frac{\mathrm{d} A}{\mathrm{~d} t}=R^{10} V_{3} \Omega_{5} \int_{0}^{z \infty} \frac{h(z) \mathrm{d} z}{z}\left(\frac{1}{\gamma^{2}} \phi\left(\frac{r_{0}}{z}\right)^{4} Z+g_{s} \chi B E\right), \\
& Z=\left(1-\sqrt{\left(1+\frac{\left.\gamma^{2}\left[\left(\frac{z^{2} \partial_{z} \phi}{r_{0}}\right)^{2}\right)^{2}-E^{2}\right]}{\phi\left(\frac{r_{0}}{z}\right)^{4}}\right)\left(1+\frac{\gamma^{2} B^{2}}{\phi\left(\frac{r_{0}}{z}\right)^{4}}\right)}\right) .
\end{aligned}
$$

It has been demonstrated that the mass of the BPS solution for this geometry is $M=4 \pi Q^{2} / r_{0}$ [35]. So, we can write $M=4 \pi v Q$, and $r_{0}=Q / v$, and we obtain

$$
\frac{\mathrm{d} \Delta \mathcal{C}_{W}}{\mathrm{~d} t} \approx \frac{0.92040 M}{\pi \hbar} \leq \frac{2 M}{\pi \hbar},
$$

where the chemical potential $v$ is defined through the coupling constant $M_{W}=g v$. Here we applied numerical techniques to obtain this holographic bound. So, we have demonstrated that, for a configuration of D3-branes, the holographic complexity from the action also satisfies an interesting holographic bound.

In this paper, we analyzed certain holographic bounds for D3-brane configurations. We analyzed the regularization of the information theoretical quantities dual to such a configuration to obtain such bounds. It may be noted that there are other interesting brane geometries in string theory. It would be interesting to calculate the holographic complexity, holographic entanglement entropy, and fidelity susceptibility for such branes. It might be possible to analyze such holographic bounds for other branes, and geometries that occur in string theory. In fact, the argument used for obtaining the relation between the holographic entanglement entropy and 
holographic complexity of a D3-brane can easily be generalized to other geometries. Thus, it would be interesting to analyze if this bound holds for other branes in string theory. In fact, even in M-theory, there exist M2-branes and M5-branes, and such quantities can be calculated for such branes. It may be noted that recently, the superconformal field theory dual to M2-branes has also been obtained, and it is a bi-fundamental Chern-Simons-matter theory called the ABJM theory [36-38]. A holographic dual to the ABJM theory with un-quenched massive flavors has also been studied [39]. It is also possible to mass-deform the ABJM theory [40], and the holographic entanglement entropy for the mass-deformed ABJM theory has been analyzed using the AdS/CFT correspondence [41]. The holographic complexity for this theory can be calculated using the same minimum surface, and the fidelity susceptibility for this theory can be calculated using the maximum volume which ends on the time slice at the boundary. It would be interesting to analyze if such a bound exists for the M2-branes. It would also be of interest to perform a similar analysis for the ABJ theory. It may be noted that the fidelity susceptibility has been used for analyzing the quantum phase transitions in condensed matter systems [42-44]. So, it is possible to holographically analyze the quantum phase transitions using this proposal. It would also be interesting to analyze the consequences of this bound on the quantum phase transition in condensed matter systems.

Acknowledgements SB is supported by the Comisión Nacional de Investigación Científica y Tecnológica (Becas Chile Grant No. 72150066).

Open Access This article is distributed under the terms of the Creative Commons Attribution 4.0 International License (http://creativecomm ons.org/licenses/by/4.0/), which permits unrestricted use, distribution, and reproduction in any medium, provided you give appropriate credit to the original author(s) and the source, provide a link to the Creative Commons license, and indicate if changes were made.

Funded by SCOAP ${ }^{3}$.

\section{References}

1. L. Borsten, D. Dahanayake, M.J. Duff, H. Ebrahim, W. Rubens, Phys. Rep. 471, 113 (2009)

2. L. Borsten, D. Dahanayake, M.J. Duff, W. Rubens, H. Ebrahim, Phys. Rev. Lett. 100, 251602 (2008)

3. M. Fujita, W. Li, S. Ryu, T. Takayanagi, JHEP 0906, 066 (2009)
4. J.M. Vilaplana, Nucl. Phys. B 888, 1 (2014)

5. H.C. Chang, A. Karch, C.F. Uhlemann, JHEP 1409, 110 (2014)

6. X. Wu, Phys. Rev. D 90, 066008 (2014)

7. G. 't Hooft. arXiv:gr-qc/9310026

8. L. Susskind, J. Math. Phys. 36, 6377 (1995)

9. J.M. Maldacena, Adv. Theor. Math. Phys. 2, 231 (1998)

10. A. Strominger, C. Vafa, Phys. Lett. B 379, 99 (1996)

11. J.M. Maldacena, JHEP 0304, 021 (2003)

12. S. Ryu, T. Takayanagi, Phys. Rev. Lett. 96, 181602 (2006)

13. V.E. Hubeny, M. Rangamani, T. Takayanagi, JHEP 0707, 062 (2007)

14. T. Albash, C.V. Johnson, JHEP 05, 079 (2012)

15. Y. Ling, P. Liu, C. Niu, J.P. Wu, Z.Y. Xian, JHEP 04, 114 (2016)

16. F. Barahona, J. Phys. A 15, 3241 (1982)

17. M. Troyer, U.J. Wiese, Phys. Rev. Lett. 94, 170201 (2005)

18. J. Grunenberg, Phys. Chem. Chem. Phys. 13, 10136 (2011)

19. M. Stanowski, Complicity 2, 78 (2011)

20. S.W. Hawking, M.J. Perry, A. Strominger, Phys. Rev. Lett. 116, 231301 (2016)

21. L. Susskind, Fortsch. Phys. 64, 24 (2016)

22. D. Stanford, L. Susskind, Phys. Rev. D 90(12), 126007 (2014)

23. D. Momeni, S.A.H. Mansoori, R. Myrzakulov, Phys. Lett. B 756, 354 (2016)

24. M. Miyaji, T. Numasawa, N. Shiba, T. Takayanagi, K. Watanabe, Phy. Rev. Lett. 115, 261602 (2015)

25. D. Carmi, R.C. Myers, P. Rath. arXiv:1612.00433 [hep-th]

26. M. Alishahiha, Phys. Rev. D 92, 126009 (2015)

27. A.R. Brown, D.A. Roberts, L. Susskind, B. Swingle, Y. Zhao, Phys. Rev. Lett. 116, 191301 (2016)

28. A.R. Brown, D.A. Roberts, L. Susskind, B. Swingle, Y. Zhao, Complexity, action and black holes. Phys. Rev. D 93, 086006 (2016)

29. A. Reynolds, S.F. Ross. arXiv: 1612.05439 [hep-th]

30. P. Kraus, F. Larsen, S.P. Trivedi, JHEP 9903, 003 (1999)

31. J. Bhattacharya, M. Nozaki, T. Takayanagi, T. Ugajin, Phys. Rev. Lett. 110(9), 091602 (2013)

32. D. Momeni, M. Raza, H. Gholizade, R. Myrzakulov, Int. J. Theor. Phys. 55(11), 4751 (2016)

33. S. Lloyd, Nature 406, 1047 (2000)

34. R.G. Cai, S.M. Ruan, S.J. Wang, R.Q. Yang, R.H. Peng, JHEP 1609, 161 (2016)

35. J.H. Schwarz, JHEP 1407, 136 (2014)

36. O. Aharony, O. Bergman, D.L. Jafferis, J. Maldacena, JHEP 0810 , 091 (2008)

37. J. Bagger, N. Lambert, S. Mukhi, C. Papageorgakis, Phys. Rept. 527, 1 (2013)

38. M. Faizal, Europhys. Lett. 98, 31003 (2012)

39. Y. Bea, E. Conde, N. Jokela, A.V. Ramallo, JHEP 12, 033 (2013)

40. M. Tierz, Phys. Rev. D 93, 126003 (2016)

41. K.K. Kim, O.K. Kwon, C. Park, H. Shin, Phys. Rev. D 90, 126003 (2014)

42. H.T. Quan, Z. Song, X.F. Liu, P. Zanardi, C.P. Sun, Decay of loschmidt echo enhanced by quantum criticality. Phys. Rev. Lett. 96, 140604 (2006)

43. P. Zanardi, N. Paunkoviic, Phys. Rev. E 74, 031123 (2006)

44. P. Zanardi, P. Giorda, M. Cozzini, Phys. Rev. Lett. 99, 100603 (2007) 